\title{
AVALIAÇÃO ECONÔMICA E EDAFOCLIMÁTICA DE REGIÕES BRASILEIRAS PARA O PLANTIO DE MOGNO AFRICANO - UMA ABORDAGEM PELOS MÉTODOS ORDINAIS: ARGUS E SAPEVO-M-NC
}

\author{
Sérgio Mitihiro do Nascimento Almeida (UFF) sergiomaeda@ gmail.com.br \\ Igor Pinheiro de Araujo Costa (UFF) costa_igor@id.uff.br \\ Carlos Francisco Simões Gomes (UFF) cfsg1@bol.com.br \\ Marcos dos Santos (IME) marcosdossantos_doutorado_uff@yahoo.com.br \\ Ivan Suarez da Mota
}

\section{Resumo}

Dados do Instituto de Pesquisas Espaciais (INPE), mostram que em 2019, o desmatamento no Brasil consumiu cerca de 1.218.708 hectares de vegetação nativa, onde um dos fatores responsáveis, deve-se à exploração de madeira considerada nobre. Dentre as medidas adotadas para mitigar o desmatamento, o plantio de florestas comerciais apresenta-se com uma importante alternativa, aumentando a oferta de madeiras no mercado, contribuindo assim, para reduzir a pressão de exploração sobre as matas nativas. É importante ressaltar que, cada espécie possui peculiaridades em relação ao seu cultivo, além disso, antes de iniciar o plantio, o produtor deve considerar os fatores econômicos associados à atividade. Diante do exposto, este artigo visa selecionar por meio dos métodos ordinais de auxílio multicritério à decisão: ARGUS e SAPEVO-M-NC, as regiões do país mais adequadas para o plantio de mogno africano da espécie ivorensis. O estudo foi realizado considerando sete regiões de diferentes estados, sendo avaliadas à luz de atributos naturais e econômicos por profissionais do setor agropecuário. Os resultados obtidos pelos métodos, indicam que os fatores climáticos possuem maior relevância para os avaliadores e apresentam uma ordenação das alternativas, alinhada com a atual conjuntura da atividade no país.

Palavras-Chaves: (Pesquisa Operacional; Sustentabilidade; Métodos Ordinais) 


\section{Introdução}

Segundo aponta o Projeto de mapeamento anual da cobertura e uso do solo no Brasil (MapBiomas), no seu relatório de 2019, o desmatamento no país consumiu 1.218.708 hectares (ha) de vegetação nativa. Desse total, mais de $60 \%$ ocorreu na Amazônia, que computou cerca de 770.000 ha devastados. Ainda, de acordo com o relatório, os biomas da Amazônia e do Cerrado representaram juntos $96,7 \%$ da área desmatada detectada, sendo que os estados do Pará, Mato Grosso e Amazonas, responderam por mais da metade deste total (AZEVEDO et al., 2020).

Além das medidas de controle, monitoramento e fiscalização ambiental, no intuito de coibir o desmatamento ilegal, há outras que visam: a recuperação de áreas degradadas por meio de reflorestamento da mata nativa; o manejo sustentável das florestas por meio de iniciativas de conservação; o uso sustentável da sua biodiversidade, dentre outras (LEMOS; SILVA, 2011). Dentre as medidas para mitigar o desmatamento florestal, bem como de proporcionar um maior desenvolvimento social, o plantio de florestas comerciais se apresenta como uma alternativa importante, pois, além do potencial de desenvolvimento econômico da região onde é inserida, possui um forte viés ambiental, uma vez que participa aumentando a oferta de madeiras no mercado, contribuindo assim, para diminuir a pressão de exploração sobre as espécies nativas.

Cada espécie possui peculiaridades com relação ao seu cultivo, no que diz respeito ao índice pluviométrico, temperatura média do local, tipo de solo, resistência a pragas, doenças, etc. Além desses fatores, o produtor deve considerar os aspectos econômicos associados ao investimento, tais como: a compra do terreno e facilidades logísticas existentes na região que possam contribuir para o escoamento da sua produção. Dado o exposto, a área a ser escolhida para o plantio deve ser criteriosamente avaliada antes do agricultor tomar uma decisão e iniciar a sua cultura.

Para Gomes e Gomes (2019), os métodos de apoio multicritério à decisão (AMD) são técnicas de assessoramento ao agente decisor para a tomada de decisões sobre problemas complexos, selecionando, avaliando ou ordenando alternativas mediante diferentes pontos de vista e dentro de critérios pré-estabelecidos.

Com base no exposto, este artigo tem por objetivo selecionar, mediante a utilização dos métodos ordinais de apoio multicritério à decisão (AMD): ARGUS e SAPEVO-M-NC, as regiões do país mais adequadas para o plantio do mogno africano do gênero Khaya e espécie ivorensis. Na 
análise, foram consideradas sete regiões de diferentes estados, onde há registros de plantio da espécie em questão: Rondônia, Tocantins, Goiás, Bahia, Espírito Santo, São Paulo e Minas Gerais, sendo avaliadas por três profissionais do setor agropecuário à luz dos seguintes critérios: Índice Pluviométrico, Temperatura média anual, Média do Valor Total do Imóvel (VTI/ha) e Infraestrutura logística para o escoamento da produção. Os resultados obtidos corroboram com o que vem ocorrendo atualmente, onde o estado de Roraima vem se destacando como um dos maiores produtores de Mogno Africano do Brasil.

\section{Metodologia}

A Figura 1 apresenta em resumo, as etapas utilizadas na pesquisa:

Figura 1 - Fluxograma da metodologia de pesquisa

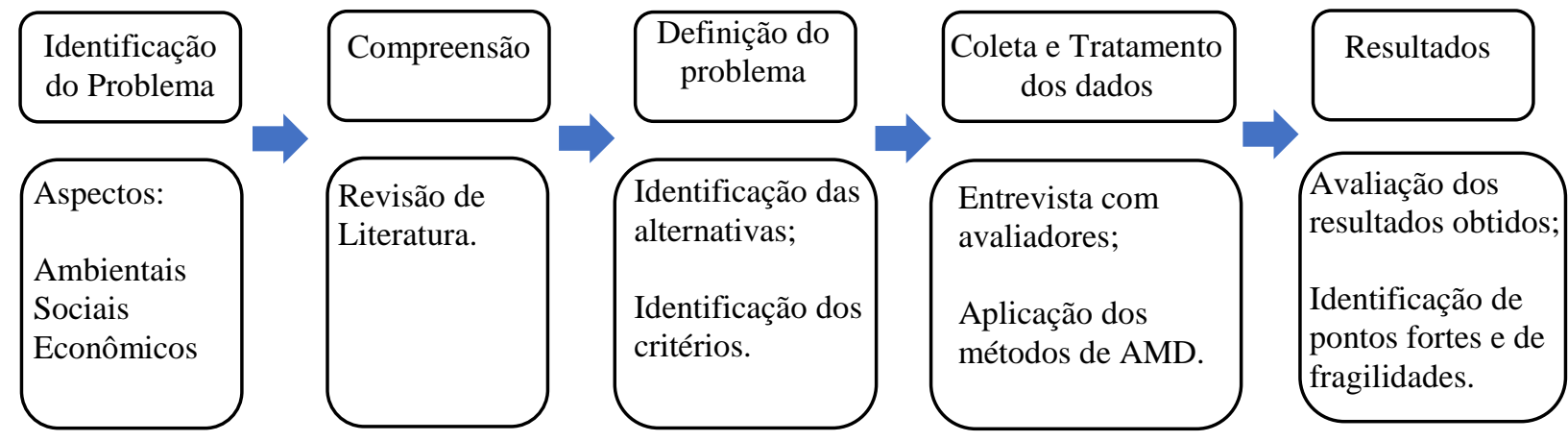

Fonte: Autores (2021)

\section{Referencial Teórico}

As espécies do gênero Khaya, comumente conhecido como mogno africano, é uma árvore de madeira dura pertence à família Meliaceae (SILVA et al., 2020). É originário de várias regiões africanas e composto por um importante grupo de espécies de elevado potencial madeireiro, sendo as Khaya anthotheca, Khaya grandifoliola, Khaya ivorensis e Khaya senegalensis, as de maior interesse comercial (REIS et al., 2019). No Brasil, os primeiros plantios se iniciaram na década de 70, na região norte e a crescente demanda pela madeira está levando a novos investimentos em plantios comerciais em todo o país, sendo a espécie ivorenses a mais difundida no território nacional (RIBEIRO et al., 2017). 


\subsection{A espécie Khaya Ivorensis}

A espécie tem sua origem em países da costa oeste da África, os quais apresentam características edafoclimáticas semelhantes a algumas regiões brasileiras, o que pode explicar a adaptação fisiológica da espécie no Brasil (REIS et al., 2019). A Khaya ivorenses possui rápido crescimento, madeira de elevada porcentagem de cerne e devidamente beneficiada, possui alta cotação no mercado internacional (RIBEIRO et al., 2017). No Brasil, a idade média para o corte raso (corte final) encontra-se entre 15 à 20 anos, com produtividade média de 400 m³/há (metros cúbicos por hectare) (COSTA et al., 2013; REIS et al., 2019).

\subsubsection{Zoneamento edafoclimático do mogno-africano (Khaya ivorensis) no Brasil}

A área de ocorrência natural da espécie engloba regiões tropicais úmidas de baixa altitude da África Ocidental. De acordo com Ribeiro et al. (2017), “O zoneamento edafoclimático é fundamental no balizamento para as tomadas de decisões, uma vez que avalia a compatibilidade entre espécies cultivadas e o ambiente a ser inserida, de modo a caracterizar e delimitar regiões com maior ou menor aptidão, ou seja, com melhor adaptação da espécie". Para o zoneamento edafoclimático no Brasil, foram observadas as características de clima e de solo dos países africanos que apresentam as maiores taxas de ocorrência natural da espécie.

A Figura 2 apresenta as áreas de aptidão edafoclimática para o cultivo da espécie no país.

Figura 2 - Áreas de aptidão edafoclimática da Khaya Ivorensis no Brasil

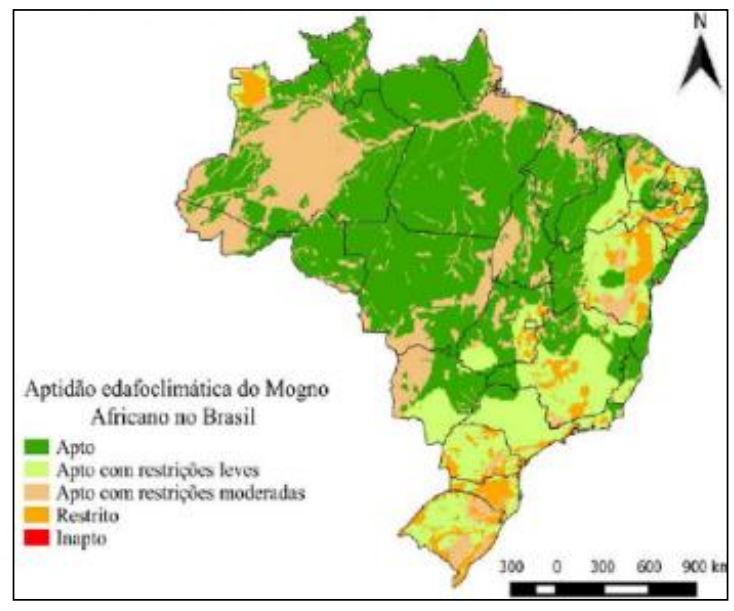

Fonte: RIBEIRO et al.(2017) 


\subsection{Materiais e Métodos}

Baseado no mapa da Figura 3, para a análise em questão, foram selecionadas para a avaliação, as seguintes áreas: Boa Vista -RR, Formoso do Araguaia - TO, Bonfinópolis - GO, Valença BA, Linhares - ES, Salmourão - SP e Pompeu - MG, as quais foram avaliadas com relação ao: índice pluviométrico, temperatura média, Média do Valor Total do Imóvel (VTI/ha) e infraestrutura logística presente na região para o escoamento da produção, os quais são apresentados abaixo:

Temperatura média (C1): De acordo com Reis et al. (2019), a faixa de temperatura média apta para o plantio, situa-se entre $18^{\circ} \mathrm{C}$ e $35^{\circ} \mathrm{C}$. As regiões que apresentam temperaturas fora desta faixa são consideradas inaptas. A temperatura média de $27,5^{\circ} \mathrm{C}$ foi considerada como ótima para o plantio da espécie em questão, assim, quanto mais próxima dessa, melhor o desempenho da alternativa;

Precipitação pluvial (C2): São consideradas aptas ao plantio, as regiões com intensidades anuais na faixa entre $830,00 \mathrm{~mm}$ e $3.000,00 \mathrm{~mm}$. Os países africanos que apresentam a maior ocorrência natural da espécie, possuem índice médio de pluviosidade anual compreendido entre 1600 e 2500mm (REIS et al., 2019). Para este critério, considerou-se que as melhores alternativas se situam mais próximo da faixa de pluviosidade de ocorrência natural da espécie.

Média do Valor Total do Imóvel (VTI/ha) (C3): Critério monotônico de custo, o qual deseja-se minimizar. Possui contribuição significativa para a definição do local do investimento, sendo a compra da terra para o cultivo, um dos custos mais elevados no projeto. Para a avaliação deste critério, foram considerados os dados gerados pelos Relatórios de Análise de Mercados de Terras (RAMT) e pelas Planilhas de Preços Referenciais de Terra (PPR) de cada local avaliado. Infraestrutura logística presente na região para o escoamento da produção (C4): Para a avaliação deste critério, considerou-se a quantidade de vias terrestres e a existência de vias fluviais nas proximidades da região de plantio, sendo considerada um critério monotônico de lucro, sendo tanto melhor, quanto maior a oferta de alternativas.

A Tabela 1 apresenta em resumo os dados das alternativas em questão: 
Tabela 1 - Dados edafoclimáticos e de capacidade logística das cidades analisadas

\begin{tabular}{lllll}
\hline \multicolumn{1}{c}{ Alternativas } & \multicolumn{5}{c}{ Critérios } \\
\hline & $\mathbf{C}_{1}$ (TM) & $\mathbf{C}_{2}$ (PM) & C3 (VTI / ha) & C4 (ILPR) \\
\hline $\mathrm{A}_{1}$ - Boa Vista - RR & $27.2^{\circ} \mathrm{C}$ & $1534 \mathrm{~mm}$ & $\mathrm{R} \$ 1.901,70$ & BR-174, BR-401, RR-205 e Rio Branco \\
$\mathrm{A}_{2}$ - Pompeu - MG & $22.7^{\circ} \mathrm{C}$ & $1239 \mathrm{~mm}$ & $\mathrm{R} \$ 7.427,21$ & MG-420, MG-164 e MG-060 \\
$\mathrm{A}_{3}$ - Bonfinópolis - GO & $23^{\circ} \mathrm{C}$ & $1365 \mathrm{~mm}$ & $\mathrm{R} \$ 22.816,34$ & GO-010 \\
$\mathrm{A}_{4}$ - Formoso do Araguaia -TO & $25,1^{\circ} \mathrm{C}$ & $1819 \mathrm{~mm}$ & $\mathrm{R} \$ 4.613,86$ & BR-226, TO-222, TO-424 e Rio Tocantis \\
$\mathrm{A}_{5}$ - Linhares - ES & $24^{\circ} \mathrm{C}$ & $1267 \mathrm{~mm}$ & $\mathrm{R} \$ 23.124,83$ & ES-248, ES-356, ES-358 e BR-101 \\
$\mathrm{A}_{6}$ - Salmourão - SP & $25,3^{\circ} \mathrm{C}$ & $1340 \mathrm{~mm}$ & $\mathrm{R} \$ 21.672,78$ & SP-294, SP-300, SP-457 e BR-267 \\
$\mathrm{A}_{7}$ - Valença - BA & $24,7^{\circ} \mathrm{C}$ & $1473 \mathrm{~mm}$ & $\mathrm{RS} 8.456,16$ & BA-001 e BA-542 \\
\hline
\end{tabular}

Fonte: Autores (2021)

Para a avaliação da importância dos critérios e do desempenho das alternativas, foram realizadas entrevistas com três profissionais do setor da agropecuário, todos com interesse no plantio de florestas como alternativa para a diversificação dos seus investimentos. De posse dos dados compilados das regiões em estudo, os métodos ARGUS e SAPEVO-M-NC, foram aplicados, a fim de apoiar a avaliação das melhores áreas para o plantio.

\subsection{O método ARGUS}

O Método ARGUS, um acrônimo para (Achieving Respect for Grades by Using ordinal Scales Only), é um método ordinal, baseado na idéia geral de sobreclassificação e que visa a problemática de escolha. Nele, as modelagens de preferência dependerão da natureza do critério, se quantitativo ou qualitativo. Inicialmente, o decisor deve realizar a modelagem de preferências do desempenho das alternativas nos critérios qualitativos, que é realizada de forma direta. Eles são comparados paritariamente, mediante as classificações ordinais: Indiferença, preferência pequena, preferência moderada, preferência forte e preferência muito forte, dando origem a uma matriz de desempenho. Para os critérios quantitativos, o decisor deve realizar a modelagem de preferências distribuindo os valores em escalas de intervalo ou de razão, onde cada uma estará associada a uma classificação ordinal. O grau de importância dos critérios é obtido de forma direta, mediante uma classificação ordinal de cinco pontos: Sem importância, Pequena importância, Moderadamente importante, Muito Importante e Extremamente importante. Após o decisor realizar as modelagens de preferência dos desempenhos e da importância dos critérios, as alternativas são comparadas paritariamente em cada critério e os resultados obtidos são combinados, dando origem à classe de desempenho obtido por cada uma, possibilitando assim verificar as relações de sobreclassificação entre as alternativas (KEYSER; PEETERS, 1994; MARTEL; MATARAZZO, 2005). 


\subsubsection{Aplicação do método}

A Tabela 2, apresenta a avaliação de desempenho das alternativas de acordo com os julgamentos dos avaliadores:

Tabela 2 -Desempenho das alternativas de acordo com os julgamentos dos avaliadores

\begin{tabular}{lcccc}
\hline \multicolumn{1}{c}{ ALTERNATIVAS } & C $_{1}$ (TM) & $\mathbf{C}_{2}(\mathbf{P M})$ & $\mathbf{C}_{3}$ (VTI / ha) & C $_{4}$ (ILPR) \\
\hline $\mathrm{A}_{1}$ - Boa Vista - RR & Muito Boa & Boa & $\mathrm{R} \$ 1.901,70$ & Muito Boa \\
$\mathrm{A}_{2}$ - Pompeu - MG & Muito Ruim & Ruim & $\mathrm{R} \$ 7.427,21$ & Média \\
$\mathrm{A}_{3}$ - Bonfinópolis - GO & Muito Ruim & Média & $\mathrm{R} \$ 22.816,34$ & Muito Ruim \\
$\mathrm{A}_{4}$ - Formoso do Araguaia -TO & Boa & Muito Boa & $\mathrm{R} \$ 4.613,86$ & Muito Boa \\
$\mathrm{A}_{5}$ - Linhares - ES & Ruim & Ruim & $\mathrm{R} \$ 23.124,83$ & Boa \\
$\mathrm{A}_{6}$ - Salmourão - SP & Boa & Média & $\mathrm{R} \$ 21.672,78$ & Boa \\
$\mathrm{A}_{7}$ - Valença - BA & Média & Boa & R $8.456,16$ & Ruim \\
\hline
\end{tabular}

Fonte: Autores (2021)

A Tabela 3, apresenta como exemplo, a modelagem de preferências das alternativas do critério $\mathrm{C}_{1}$, de acordo com os julgamentos dos avaliadores:

Tabela 3 - Modelagem de desempenho das alternativas no critério C1 - Temperatura Média

\begin{tabular}{cccccc}
\hline & Muito Ruim & Ruim & Média & Boa & Muito Boa \\
\hline Muito Ruim & indiferente & & & & \\
Ruim & Pref. Pequena & indiferente & & & \\
Média & Pref. Forte & Pref. Moderada & indiferente & & \\
Boa & Pref. Muito Forte & Pref. Muito Forte & Pref. Moderada & indiferente & \\
Muito Boa & Pref. Muito Forte & Pref. Muito Forte & Pref. Moderada & Pref. Pequena & indiferente \\
\hline
\end{tabular}

Fonte: Autores (2021)

A Tabela 4, apresenta a modelagem da importância dos critérios, de acordo com os julgamentos dos avaliadores:

Tabela 4 - Modelagem da importância dos critérios

\begin{tabular}{ll}
\hline \multicolumn{1}{c}{ Classificação } & Critérios \\
\hline Sem importância & \\
Pequena Importância & $\mathrm{C}_{3}-$ Valor Total do Imóvel \\
Moderadamente Importante & $\mathrm{C}_{4}-$ Infraestrutura Logistica \\
Muito Importante & $\mathrm{C}_{2}-$ Pluviosidade Média \\
Extremamente Importante & $\mathrm{C}_{1}-$ Temperatura Média \\
\hline
\end{tabular}

Fonte: Autores (2021)

A Tabela 5 apresenta a combinação das modelagens de desempenho das alternativas e importâncias dos critérios (CPW). 
Tabela 5 - Combinação do desempenho das alternativas e da importância dos critérios

\begin{tabular}{cl}
\hline Classes & \multicolumn{1}{c}{ Combinaçào das Preferências } \\
\hline 1 & Preferência Muito Forte - Extremamente Importante \\
\hline 2 & Preferência Muito Forte - Muito Importante / Preferência Forte - Extremamente Importante \\
\hline 3 & $\begin{array}{l}\text { Preferência Muito Forte - Moderadamente Importante / Preferência Forte - Muito Importante } \\
\text { Preferência Moderada - Extremamente Importante }\end{array}$ \\
\hline 4 & $\begin{array}{l}\text { Preferência Muito Forte - Pequena Importância / Preferência Forte - Moderadamente Importante } \\
\text { Preferência Moderada - Muito Importante / Preferência Pequena - Extremamente Importante }\end{array}$ \\
\hline 5 & $\begin{array}{l}\text { Preferência Muito Forte - Sem Importância / Preferência Forte - Pequena Importância } \\
\text { Preferência Moderada - Moderadamente Importante / Preferência Pequena - Muito Importante }\end{array}$ \\
\hline 6 & $\begin{array}{l}\text { Preferência Forte - Sem Importância / Preferência Moderada - Pequena Importância } \\
\text { Preferência Pequena - Moderadamente Importante }\end{array}$ \\
\hline 7 & Preferência Moderada - Sem Importância / Preferência Pequena - Pequena Importância \\
\hline 8 & Preferência Pequena - Sem Importância \\
\hline
\end{tabular}

Fonte: Autores (2021)

Como resultado, verificamos a ordenação final das preferências das alternativas:

$\mathrm{A} 1>\mathrm{A} 4>\mathrm{A} 6>\mathrm{A} 7>\mathrm{A} 5>\mathrm{A} 2>\mathrm{A} 3$. Pela proposta do método, a alternativa A1 seria a melhor escolha.

\subsection{O método SAPEVO-M-NC}

Derivado do método ordinal SAPEVO-M (Simple Aggregation of Preferences Expressed by Ordinal Vectors - Multi Decision Makers) (GOMES et al., 2020). O método SAPEVO-M-NC (Simple Aggregation of Preferences Expressed by Ordinal Vectors - Non Compensatory Multi Decision Makers), consiste em um método de natureza ordinal, não-compensatório, que visa a problemática de ordenação $(\mathrm{P} \gamma)$ e com possibilidade de avaliação por múltiplos decisores. No método, a avaliação do desempenho das alternativas é realizada de forma direta, não havendo a necessidade de se realizar comparações paritárias entre as alternativas a fim de se obter a modelagem de preferências entre elas, resultando em uma redução substancial do esforço cognitivo por parte do(s) decisor(es). O método permite ainda, avaliar a taxa de sobreclassificação entre as alternativas. Esta informação possibilita não só ao Decision Maker (DM) verificar a relação de dominância, como também, permite identificar em termos percentuais, o quanto uma alternativa supera a outra;

Diferente da Teoria de Decisão Clássica, que considera basicamente duas relações de preferência, supostamente transitivas, designadas por Indiferença (I) e por preferência estrita (P), este método baseia-se no Sistema Fundamental de Relações de Preferências (SFRP), incorporando também a relação de preferência fraca (Q). De acordo com Gomes e Gomes 
(2002), há várias razões pelas quais os pesquisadores buscam evitar o tipo de modelagem que se baseia no axioma de comparabilidade completa e transitividade entre as alternativas, das quais, pode-se citar o fato de que o decisor não possuir todas as informações que o permita optar por uma das alternativas e ao forçar uma relação de preferência estrita, ou de indiferença, poderia levar a assumir erros arbitrários e não coerentes.

O método pode ser divido em sete etapas:

Etapa 1 - Estruturação da matriz de decisão:

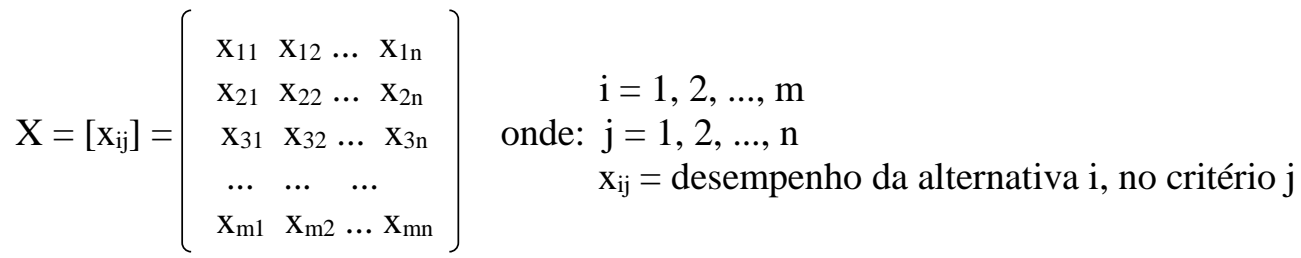

Etapa 2 - Transformação ordinal das preferências dos DM em cada critério, as quais são agregadas ao fim desta etapa, dando origem a um vetor $\left(\mathrm{V}_{\mathrm{i}}\right)$, representando os pesos dos critérios (GOMES et al., 2020).

Sejam $c_{i}$ e $c_{j}$, dois critérios dentro de um conjunto de critérios $C=\left\{c_{1}, c_{2}, c_{3} \ldots c_{i}, c_{j}\right\} . O$ grau de preferência entre eles é dado por: $\delta\left(c_{i} c_{j}\right)$, onde:

$$
\begin{aligned}
& \delta\left(c_{i} c_{j}\right)=1 \leftrightarrow c_{i} \approx c_{j}\left(c_{i} \text { é tão importante quanto } c_{j}\right) \\
& \delta\left(c_{i} c_{j}\right)>1 \leftrightarrow c_{i}>c_{j}\left(c_{i} \text { é mais importante quanto } c_{j}\right) \\
& \delta\left(c_{i} c_{j}\right)<1 \leftrightarrow c_{i}<c_{j}\left(c_{i} \text { é menos importante quanto } c_{j}\right)
\end{aligned}
$$

A Tabela 6 apresenta a relação da importância relativa entre os critérios:

Tabela 6 - Importância relativa entre os critérios

\begin{tabular}{ccc}
\hline Escala 1 & Representação Verbal & Escala 2 \\
\hline 1 & Absolutamente menos importante & -3 \\
$\prec 1$ & Muito menos importante & -2 \\
$\prec 1$ & Menos importante & -1 \\
1 & Igualmente importante & 0 \\
$\succ 1$ & Mais importante & 1 \\
$\nsucc 1$ & Muito mais importante & 2 \\
$\star 1$ & Absolutamente mais importante & 3 \\
\hline
\end{tabular}

Fonte: Autores (2021)

Seja $\mathrm{D}$ um conjunto de agentes decisores, $\mathrm{D}=\left\{\mathrm{DM}_{1}, \mathrm{DM}_{2}, \ldots, \mathrm{DM}_{\mathrm{k}}, \ldots \mathrm{DM}_{\mathrm{n}}\right\}$ que expressam suas opiniões sobre a importância relativa dos critérios envolvidos. Essas preferências dão 
origem a matriz de preferências $\mathrm{MDM}_{\mathrm{k}}$. A relação entre as duas escalas da Tabela permite a transformação da matriz (1) em (2):

$\mathrm{MDM}_{\mathrm{k}}=\left[\delta\left(\mathrm{c}_{\mathrm{i}} \mathrm{c}_{\mathrm{j}}\right)\right]$, em um vetor coluna $\left[\mathrm{V}_{\mathrm{i}}\right]$, onde:

$\mathrm{V}_{\mathrm{i}}=\sum_{j=1}^{m} \delta(\mathrm{ci} \mathrm{cj})(\mathrm{i}=1, \ldots, \mathrm{m} \mathrm{e} \mathrm{k}=1, \ldots, \mathrm{n})$

Após gerar o vetor $\mathrm{V}_{\mathrm{i}}$, os seus elementos $\mathrm{a}_{\mathrm{ij}}$ são normalizados de acordo com (3):

$\mathrm{v}=\left(\mathrm{a}_{\mathrm{ij}}-\min \mathrm{a}_{\mathrm{ij}}\right) /\left(\max \mathrm{a}_{\mathrm{ij}}-\min \mathrm{a}_{\mathrm{ij}}\right)$

Dando origem ao vetor de preferências do $\mathrm{DM}_{\mathrm{k}}$. Ocorrendo valores nulos nesta etapa, estes são substituídos por $1 \%$ do segundo menor valor obtido. Após todos os " $\mathrm{k}$ " DM's realizarem suas avaliações, os vetores normalizados são somados, dando origem ao vetor de pesos que expressa a importância dos critérios (GOMES et al., 2020).

Etapa 3 - Classificação ordinal $\left(\Theta_{\mathrm{ij}}\right)$ do desempenho das alternativas:

Nesta etapa, cada DM atribui as classificações relativas aos desempenhos das alternativas em cada critério (Tabela 7), as quais são relacionadas às suas faixas de classificação $\mathrm{g}_{(\mathrm{ij})}$. Após todos os " $n$ " DM realizarem suas avaliações, é obtida a média aritmética $\mu_{(\mathrm{ij})}$ das faixas de classificação dos desempenhos das alternativas em cada critério.

Tabela 7 - Classificação do desempenho das alternativas em cada critério

\begin{tabular}{cc}
\hline $\begin{array}{c}\text { Classificação ordinal }\left(\Theta_{\mathrm{ij}} \text { ) do desempenho }\right. \\
\text { da alternativa i no critério j }\end{array}$ & Faixa de Classificação $\mathrm{g}_{(\mathrm{ij})}$ \\
\hline Excelente $(\mathrm{E})$ & 1 \\
Muito Bom $(\mathrm{MB})$ & 2 \\
Bom $(\mathrm{B})$ & 3 \\
Médio $(\mathrm{M})$ & 4 \\
Ruim $(\mathrm{R})$ & 5 \\
Muito Ruim (MR) & 6 \\
Péssimo (P) & 7 \\
\hline
\end{tabular}

Fonte: Autores (2021)

Etapa 4 - Obtenção das frações dos pesos dos critérios $\left(\sigma_{\mathrm{j}(\mathrm{ab})}\right)$.

Para cada critério “i”, realiza-se a comparação paritária entre as alternativas a fim de se verificar a distância relativa entre as faixas de classificação, onde (4):

$\Delta \mu_{\mathrm{j}(\mathrm{ab})}=\mu_{(\mathrm{ja})}-\mu_{(\mathrm{b})}$

Este valor, permite identificar na modelagem de preferências (Figura 3 e Tabela 8), a fração de peso do critério “j” obtida pela alternativa "a" em relação à alternativa "b" $\left(\sigma_{j}(a b)\right)$. 
Figura 3 - Função de preferência de um critério com variação linear.

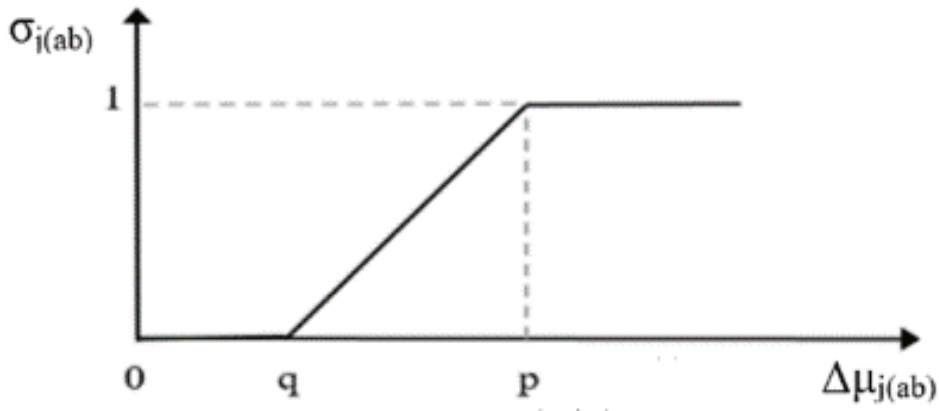

Fonte: Autores (2021)

Tabela 8 - Modelagem de preferências dos critérios

\begin{tabular}{|c|c|c|}
\hline Indiferença (I) & $\mu_{(\mathrm{ia})}-\mu_{(\mathrm{ib})} \leq 1: \sigma_{\mathrm{i}(\mathrm{ab})} \rightarrow$ & 0 \\
\hline Preferência Fraca (Q) & $1<\mu_{(\mathrm{ia})}-\mu_{(\mathrm{ib})} \leq 3: \sigma_{\mathrm{i}(\mathrm{ab})} \rightarrow$ & $\frac{\left(a_{i j}-\min a_{i j}\right)}{\left(\operatorname{máx} a_{i j}-\min a_{i j}\right)}$ \\
\hline Preferência Forte $(\mathrm{P})$ & $3<\mu_{(\mathrm{ia})}-\mu_{(\mathrm{ib})}$ & 1 \\
\hline
\end{tabular}

Fonte: Autores (2021)

Obtida pela soma ponderada dos pesos dos critérios $\left(\mathrm{W}_{\mathrm{j}}\right)$, com a fração correspondente $\left(\sigma_{\mathrm{j}(\mathrm{ab})}\right)$ verificada na modelagem de preferências (5):

$\mathrm{d}_{\mathrm{ab}}=\sum \mathrm{W}_{\mathrm{i}} \mathrm{X} \sigma_{\mathrm{i}(\mathrm{ab})}$

Etapa 6 - Cálculo da dominância absoluta $\mathrm{D}_{\mathrm{ab}}$ e Taxa de sobreclassificação $\eta_{\mathrm{ab}}$ :

A diferença entre as dominâncias relativas $d_{a b}-d_{b a}$, fornece a informação da dominância absoluta $\mathrm{D}_{\mathrm{ab}}$ entre as alternativas (6).

$\mathrm{D}_{\mathrm{ab}}=\mathrm{d}_{\mathrm{ab}}-\mathrm{d}_{\mathrm{ba}}$

Dividindo-se esse valor, pelo somatório dos pesos, obtém-se a taxa percentual de dominância absoluta entre as alternativas.

(Obs: Se $\mathrm{D}_{\mathrm{ab}}=0$, verifica-se a pontuação total obtida pelas alternativas avaliadas sobre as demais $\left(\sum \mathrm{d}_{\mathrm{aj}} \mathrm{e} \sum \mathrm{d}_{\mathrm{bj}}\right)$. Por exemplo, caso $\sum \mathrm{d}_{\mathrm{aj}}>\sum \mathrm{d}_{\mathrm{bj}}$, a alternativa "a" domina a alternativa " $\mathrm{b}$ ".

Etapa 7 - Montagem do grafo com as relações de dominância entre as alternativas.

\subsubsection{Aplicação do método SAPEVO-M-NC}


Considerando a matriz de decisão apresentada na Tabela 1, o processo se inicia com a avaliação dos atributos pelos DM's (Etapa 2). O quadro 1 apresenta como exemplo, a avaliação ordinal dos atributos pelo primeiro decisor $\left(\mathrm{DM}_{1}\right)$.

Quadro 1 -Avaliação ordinal da importância dos atributos pelo $\mathrm{DM}_{1}$

\begin{tabular}{|c|c|c|c|c|}
\hline & $\mathrm{C}_{1}(\mathrm{TM})$ & $\mathrm{C}_{2}(\mathrm{PM})$ & $\mathrm{C}_{3}$ (VTI/ ha) & $\mathrm{C}_{4}$ (ILPR) \\
\hline $\mathrm{C}_{1}(\mathrm{TM})$ & $\begin{array}{l}\text { Igualmente } \\
\text { importante }\end{array}$ & Mais importante & $\begin{array}{l}\text { Absolutamente mais } \\
\text { importante }\end{array}$ & $\begin{array}{l}\text { Muito mais } \\
\text { importante }\end{array}$ \\
\hline $\mathrm{C}_{2}(\mathrm{PM})$ & Menos importante & Igualmente importante & Muito mais importante & Mais importante \\
\hline $\mathrm{C}_{3}$ (VTI/ha) & $\begin{array}{l}\text { Absolutamente } \\
\text { menos importante }\end{array}$ & $\begin{array}{l}\text { Muito menos } \\
\text { importante }\end{array}$ & Igualmente importante & $\begin{array}{c}\text { Menos } \\
\text { importante }\end{array}$ \\
\hline $\mathrm{C}_{4}$ (ILPR) & $\begin{array}{l}\text { Muito menos } \\
\text { importante }\end{array}$ & Menos importante & Mais importante & $\begin{array}{l}\text { Igualmente } \\
\text { importante }\end{array}$ \\
\hline
\end{tabular}

Fonte: Autores (2021)

A Tabela 9, apresenta os valores relativos às avaliações ordinais do $\mathrm{DM}_{1}$, juntamente com o vetor de pesos normalizados.

Tabela 9 - Avaliação da importância dos atributos pelo $\mathrm{DM}_{1}$

\begin{tabular}{ccccccc}
\hline & $\mathrm{C}_{1}$ (TM) & $\mathrm{C}_{2}$ (PM) & $\mathrm{C}_{3}$ (VTL/ha) & $\mathrm{C}_{4}($ ILPR $)$ & $\mathrm{V}_{\mathrm{i}}$ & Vetor normalizado \\
\hline $\mathrm{C}_{1}(\mathrm{TM})$ & 0 & +1 & +3 & +2 & 6 & 1 \\
$\mathrm{C}_{2}$ (PM) & -1 & 0 & +2 & +1 & 2 & 0,666667 \\
$\mathrm{C}_{3}$ (VTL/ha) & -3 & -2 & 0 & -1 & -6 & 0,003333 \\
$\mathrm{C}_{4}$ (ILPR) & -2 & -1 & +1 & 0 & -2 & 0,333333 \\
\hline
\end{tabular}

Fonte: Autores (2021)

A Tabela 10, apresenta o valor dos pesos dos critérios, após avaliação pelos DM's.

Tabela 10 - Avaliação da importância dos critérios pelos DM’s

\begin{tabular}{ccccc}
\hline & $\mathrm{DM}_{1}$ & $\mathrm{DM}_{2}$ & $\mathrm{DM}_{3}$ & Pesos dos critérios \\
\hline $\mathrm{C}_{1}(\mathrm{TM})$ & 1 & 0,875 & 1 & 2,875 \\
$\mathrm{C}_{2}$ (PM) & 0,6667 & 1 & 0,5555 & 2,222 \\
$\mathrm{C}_{3}$ (VTI $/$ ha) & 0,0033 & 0,00875 & 0,2222 & 0,234 \\
$\mathrm{C}_{4}$ (ILPR) & 0,3333 & 0,125 & 0,0022 & 0,461 \\
\hline
\end{tabular}

Fonte: Autores (2021)

A Tabela 11, apresenta a classificação do desempenho das alternativas pelos DM's:

Tabela 11 - Classificação do desempenho das alternativas pelos DM's 


\begin{tabular}{|c|c|c|c|c|c|c|c|c|c|c|c|c|c|c|c|c|c|c|c|}
\hline \multicolumn{5}{|c|}{$\mathrm{DM}_{1}$} & \multicolumn{5}{|c|}{$\mathrm{DM}_{2}$} & \multicolumn{5}{|c|}{$\mathrm{DM}_{3}$} & \multicolumn{5}{|c|}{ Média $\mu_{(i i)}$} \\
\hline & $\mathrm{C}_{1}$ & $\mathrm{C}_{2}$ & $\mathrm{C}_{3}$ & $\mathrm{C}_{4}$ & & $\mathrm{C}_{1}$ & $\mathrm{C}_{2}$ & $\mathrm{C}_{3}$ & $\mathrm{C}_{4}$ & & $\mathrm{C}_{1}$ & $\mathrm{C}_{2}$ & $\mathrm{C}_{3}$ & $\mathrm{C}_{4}$ & & $\mathrm{C}_{1}$ & $\mathrm{C}_{2}$ & $\mathrm{C}_{3}$ & $\mathrm{C}_{4}$ \\
\hline $\mathrm{A}_{1}$ & 1 & 2 & 1 & 1 & $A_{1}$ & 1 & 3 & 1 & 1 & $\mathrm{~A}_{1}$ & 1 & 2 & 1 & 1 & $\mathrm{~A}_{1}$ & 1 & 2,33 & 1 & 1 \\
\hline $\mathrm{A}_{2}$ & 6 & 5 & 3 & 3 & $\mathrm{~A}_{2}$ & 6 & 6 & 3 & 3 & A2 & 6 & 6 & 3 & 3 & $\mathrm{~A}_{2}$ & 6 & 5,66 & 3 & 3 \\
\hline $\mathrm{A}_{3}$ & 6 & 4 & 6 & 5 & $\mathrm{~A}_{3}$ & 6 & 5 & 6 & 5 & $\mathrm{~A}_{3}$ & 6 & 5 & 5 & 6 & $\mathrm{~A}_{3}$ & 6 & 4,66 & 5,66 & 5,33 \\
\hline $\mathrm{A}_{4}$ & 3 & 1 & 2 & 1 & $\mathrm{~A}_{4}$ & 2 & 1 & 2 & 1 & $\mathrm{~A}_{4}$ & 2 & 1 & 2 & 1 & $\mathrm{~A}_{4}$ & 2,33 & 1 & 2 & 1 \\
\hline $\mathrm{A}_{5}$ & 5 & 5 & 6 & 2 & $\mathrm{~A}_{5}$ & 5 & 6 & 6 & 2 & $\mathrm{~A}_{5}$ & 4 & 6 & 5 & 2 & $\mathrm{~A}_{5}$ & 4,66 & 5,66 & 5,66 & 2 \\
\hline $\mathrm{A}_{6}$ & 3 & 4 & 6 & 2 & $\mathrm{~A}_{6}$ & 2 & 5 & 5 & 2 & $\mathrm{~A}_{6}$ & 2 & 5 & 5 & 2 & $\mathrm{~A}_{6}$ & 2,33 & 4,66 & 5,33 & 2 \\
\hline $\mathrm{A}_{7}$ & 4 & 3 & 3 & 4 & $\mathrm{~A}_{7}$ & 4 & 4 & 3 & 4 & $\mathrm{~A}_{7}$ & 3 & 4 & 3 & 4 & $\mathrm{~A}_{7}$ & 3,66 & 3,66 & 3 & 4 \\
\hline
\end{tabular}

Fonte: Autores (2021)

A Tabela 12 apresenta respectivamente, a diferença entre as avaliações dos desempenhos das

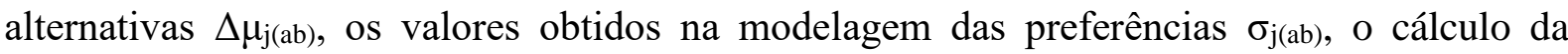
dominância relativa $d_{a b}$, a dominância absoluta $D_{a b}$ e a taxa de sobreclassificação $\eta_{a b}$.

Tabela 12 - Valores de $\Delta \mu_{\mathrm{j}(\mathrm{ab})}, \sigma_{\mathrm{j}}(\mathrm{ab}), \mathrm{d}_{\mathrm{ab}}, \mathrm{D}_{\mathrm{ab}}$ e $\eta_{\mathrm{ab}}$

\begin{tabular}{|c|c|c|c|c|c|c|c|c|c|c|c|c|c|c|c|}
\hline \multicolumn{5}{|c|}{$\Delta \mu_{j(\mathrm{ab})}=\mu_{(\mathrm{aj})}-\mu_{(\mathrm{bj})}$} & \multicolumn{4}{|c|}{$\sigma_{j(a b)}$} & \multicolumn{5}{|c|}{$d_{a b}=\sum w_{i} x \sigma_{i(a b)}$} & $D_{a b}$ & $\eta_{\mathrm{ab}}$ \\
\hline & $\mathrm{C} 1$ & $\mathrm{C} 2$ & $\mathrm{C} 3$ & $\mathrm{C} 4$ & $\mathrm{C} 1$ & $\mathrm{C} 2$ & $\mathrm{C} 3$ & $\mathrm{C} 4$ & $\mathrm{C} 1$ & $\mathrm{C} 2$ & $\mathrm{C} 3$ & $\mathrm{C} 4$ & Total & Total & Total \\
\hline $\mathrm{A} 1-\mathrm{A} 2$ & 5,00 & 3,33 & 2,00 & 2,00 & 1,00 & 1,00 & 0,50 & 0,50 & 2,88 & 2,22 & 0,12 & 0,23 & 5,44 & 5,44 & 0,94 \\
\hline $\mathrm{A} 1-\mathrm{A} 3$ & 5,00 & 2,33 & 4,67 & 4,33 & 1,00 & 0,67 & 1,00 & 1,00 & 2,88 & 1,48 & 0,23 & 0,46 & 5,05 & 5,05 & 0,87 \\
\hline A1-A4 & 1,33 & $-1,33$ & 1,00 & 0,00 & 0,17 & & 0,00 & 0,00 & 0,48 & 0,00 & 0,00 & 0,00 & 0,48 & 0,11 & 0,02 \\
\hline A1-A5 & 3,67 & 3,33 & 4,67 & 1,00 & 1,00 & 1,00 & 1,00 & 0,00 & 2,88 & 2,22 & 0,23 & 0,00 & 5,33 & 5,33 & 0,92 \\
\hline A1-A6 & 1,33 & 2,33 & 4,33 & 1,00 & 0,17 & 0,67 & 1,00 & 0,00 & 0,48 & 1,48 & 0,23 & 0,00 & 2,19 & 2,19 & 0,38 \\
\hline A1-A7 & 2,67 & 1,33 & 2,00 & 3,00 & 0,83 & 0,00 & 0,50 & 1,00 & 2,40 & 0,00 & 0,12 & 0,46 & 2,97 & 2,97 & 0,51 \\
\hline A2-A3 & 0,00 & $-1,00$ & 2,67 & 2,33 & 0,00 & - & 0,83 & 0,67 & 0,00 & 0,00 & 0,20 & 0,31 & 0,50 & 0,50 & 0,09 \\
\hline A2-A4 & $-3,67$ & $-4,67$ & $-1,00$ & $-2,00$ & - & - & - & - & 0,00 & 0,00 & 0,00 & 0,00 & 0,00 & $-5,33$ & $-0,92$ \\
\hline -A5 & $-1,33$ & 0,00 & 2,67 & $-1,00$ & - & 0,00 & 0,83 & - & 0,00 & 0,00 & 0,20 & 0,00 & 0,20 & $-0,28$ & $-0,05$ \\
\hline & $-3,67$ & $-1,00$ & 2,33 & $-1,00$ & - & - & 0,67 & - & 0,00 & 0,00 & 0,16 & 0,00 & 0,16 & $-2,72$ & $-0,47$ \\
\hline A $2-\mathrm{A} 7$ & $-2,33$ & $-2,00$ & 0,00 & 1,00 & - & - & 0,00 & 0,00 & 0,00 & 0,00 & 0,00 & 0,00 & 0,00 & $-3,03$ & $-0,52$ \\
\hline A3-A4 & $-3,67$ & $-3,67$ & $-3,67$ & $-4,33$ & - & - & - & - & 0,00 & 0,00 & 0,00 & 0,00 & 0,00 & $-5,79$ & $-1,00$ \\
\hline A $3-\mathrm{A} 5$ & $-1,33$ & 1,00 & 0,00 & $-3,33$ & - & 0,00 & 0,00 & - & 0,00 & 0,00 & 0,00 & 0,00 & 0,00 & $-0,46$ & $-0,08$ \\
\hline A3-A6 & $-3,67$ & 0,00 & $-0,33$ & $-3,33$ & - & 0,00 & - & - & 0,00 & 0,00 & 0,00 & 0,00 & 0,00 & $-3,34$ & $-0,58$ \\
\hline A3-A7 & $-2,33$ & $-1,00$ & $-2,67$ & $-1,33$ & - & - & - & - & 0,00 & 0,00 & 0,00 & 0,00 & 0,00 & $-2,19$ & $-0,38$ \\
\hline A4-A5 & 2,33 & 4,67 & 3,67 & 1,00 & 0,67 & 1,00 & 1,00 & 0,00 & 1,92 & 2,22 & 0,23 & 0,00 & 4,37 & 4,37 & 0,75 \\
\hline A4-A6 & 0,00 & 3,67 & 3,33 & 1,00 & 0,00 & 1,00 & 1,00 & 0,00 & 0,00 & 2,22 & 0,23 & 0,00 & 2,46 & 2,46 & 0,42 \\
\hline A4-A7 & 1,33 & 2,67 & 1,00 & 3,00 & 0,17 & 0,83 & 0,00 & 1,00 & 0,48 & 1,85 & 0,00 & 0,46 & 2,79 & 2,79 & 0,48 \\
\hline A5-A6 & $-2,33$ & $-1,00$ & $-0,33$ & 0,00 & - & - & - & 0,00 & 0,00 & 0,00 & 0,00 & 0,00 & 0,00 & $-1,92$ & $-0,33$ \\
\hline A5-A7 & $-1,00$ & $-2,00$ & $-2,67$ & 2,00 & - & - & - & 0,50 & 0,00 & 0,00 & 0,00 & 0,23 & 0,23 & $-1,08$ & $-0,19$ \\
\hline A6-A7 & 1,33 & $-1,00$ & $-2,33$ & 2,00 & 0,17 & - & - & 0,50 & 0,48 & 0,00 & 0,00 & 0,23 & 0,71 & 0,55 & 0,10 \\
\hline
\end{tabular}

Fonte: Autores (2021)

Mediante os resultados obtidos, verificamos as seguintes relações de dominância:

$$
\begin{aligned}
& A_{1}>\left(A_{2} ; A_{3} ; A_{4} ; A_{5} ; A_{6} ; A_{7}\right) \\
& A_{4}>\left(A_{2} ; A_{3} ; A_{5} ; A_{6} ; A_{7}\right) \\
& A_{6}>\left(A_{2} ; A_{3} ; A_{5}: A_{7}\right) \\
& A_{7}>\left(A_{2} ; A_{3} ; A_{5}\right) \\
& A_{5}>\left(A_{2} ; A_{3}\right) \\
& A_{2}>A_{3}
\end{aligned}
$$

E o grafo com as relações de dominância pode ser construído (Figura 4):

Figura 4 - Grafo representando as relações de dominância entre as alternativas.

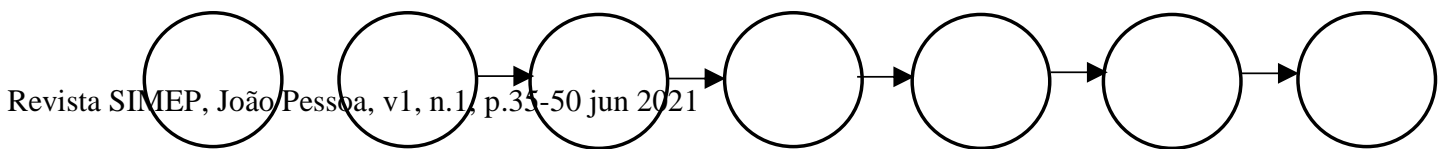




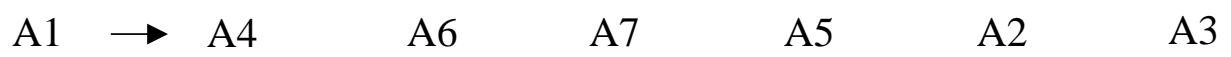

Fonte: Autores (2021)

\section{Discussão dos Resultados}

Os resultados obtidos mediante a aplicação dos métodos ordinais apontam para o mesmo direcionamento na ordenação de preferências das melhores regiões para o plantio do mogno africano. Para a avaliação dos atributos e alternativas, foram consideradas os julgamentos de profissionais ligados ao setor agropecuário, mas não necessariamente especialistas, indicando o ganho que as ferramentas da pesquisa operacional podem proporcionar à sociedade como um todo. Verifica-se que os critérios climáticos apresentaram maiores níveis de importância em relação aos econômicos, uma vez que regiões com grandes distanciamentos das condições consideradas ideais no estudo, podem inviabilizar o plantio da espécie em questão.

A avaliação das alternativas pelo método ARGUS, foi realizada baseadas nas opiniões dos profissionais consultados. Já pelo método SAPEVO-M-NC, devido a sua axiomática, ambos os profissionais do setor agropecuário tiveram seus julgamentos considerados de forma independente. Os resultados obtidos por ambos os métodos apresentaram a mesma ordenação final, entretanto, verifica-se que o esforço cognitivo exigido dos decisores na avaliação por este último é menor, facilitando de sobremaneira a sua aplicação. A possibilidade de avaliação da taxa de sobreclassificação entre as alternativas, possibilita ao(s) DM(s) uma clareza maior em seus julgamentos, uma vez que se pode verificar com qual intensidade uma alternativa domina a outra.

\section{Considerações Finais e Conclusão}

Os resultados corroboram com o que vem ocorrendo na atualidade, onde o estado de Roraima vem se destacando como um dos maiores produtores de mogno africano do Brasil. Devido à relativa facilidade de aplicação, associada a um baixo esforço cognitivo por parte dos avaliadores, verifica-se que a metodologia SAPVO-M-NC pode vir a proporcionar grandes ganhos, não só para a comunidade acadêmica, mas também para a sociedade como um todo, se apresentando como uma alternativa de ferramenta no auxílio multicritério à decisão, de carácter ordinal, não compensatório e com possibilidade de suporte a múltiplos decisores. 


\section{Referências}

AZEVEDO, T. R. et al. Relatório Anual do Desmatamento no Brasil Relatório Anual do Desmatamento no Brasil. Mapbiomas, p. 49, 2020.

COSTA, J. R. Cultivo e Manejo do Mogno Manaus - AM. Embrapa Amazônia Ocidental, 2013

GOMES, L. F. A. M., e Gomes, C. F. S. Princípios e métodos para a tomada de decisão: Enfoque multicritério (6a ed.). São Paulo: Atlas. (2019).

GOMES, Carlos Francisco Simões et al . SAPEVO-M: A Group Multicriteria Ordinal Ranking

Method. Revista Pesquisa Operacional, Rio de Janeiro, v. 40, 2020.

KEYSER, W.S.M., PEETERS, P.H.M. ARGUS - A new multiple criteria method based on the general idea of outranking. Applying Multiple Criteria Aid for Decision to Environmental Management, Kluwer Academic Publishers, Dordrecht, 1994.

LEMOS, A. L. F., SILVA, J. A. Desmatamento na Amazônia Legal: Evolução, Causas, Monitoramento e Possibilidades de Mitigação Através do Fundo Amazônia. Floresta e Ambiente, v. 18, n. 1, p. 98-108, 2011.

MARTEL J.M., MATARAZZO B., 2005. Other outranking approaches. F.J.Salvatore, G.M.Ehrgott, Eds., Multiplecriteria Decision Analysis: State of the Art Surveys. Springer, Nova York, 210-215

REIS, C. A. F.; OLIVEIRA, E. B. DE; SANTOS, A. M. Mogno-africano: atualidades e perspectivas do cultivo no Brasil, 2019.

RIBEIRO, A.; FILHO, A. C. F.; SCOLFORO, J. R. S. African Mahogany. cultivation and the increase of the activity in Brazil. Floresta e Ambiente, v. 24, p. 504-508, 2017.

SILVA, M. C. et al. Aplicação de redes neurais artificiais na cultura do mogno (Khaya spp. e Swietenia spp.). Journal of Biotechnology and Biodiversity, v. 8, n. 1, p. 017-023, 2020. 